\title{
Two-dimensional optical ring resonators based on radial Bragg resonance
}

\author{
Jacob Scheuer and Amnon Yariv \\ Department of Applied Physics, 128-95 California Institute of Technology, Pasadena, California 91125
}

Received May 1, 2003

\begin{abstract}
A Bragg-reflection-based ring resonator is proposed and analyzed. Closed-form expressions for the field and dispersion curves for radial Bragg gratings and photonic bandgap crystals are derived. The required gratings exhibit a chirped period and a varying index profile. Small bending radii and strong control over the resonator dispersion are possible by the Bragg confinement. Large free spectral range and low radiation loss are predicted theoretically. (C) 2003 Optical Society of America

OCIS codes: $130.2790,230.5750$.
\end{abstract}

In the past few years there has been a substantial increase in activity dealing with utilization of ring resonators for optical communication devices. Various ring-resonator-based applications such as modulators, ${ }^{1}$ channel drop filters, ${ }^{2}$ and dispersion compensators ${ }^{3}$ have been suggested and demonstrated. The important characteristics of the modes of ring resonators are the free spectral range (FSR) and the loss per revolution, or equivalently the $Q$ factor.

In this Letter we propose a new type of a ring resonator based on a single annular defect located between radial Bragg reflectors. We show that the modes of such structures can have a large $Q$ factor and a large FSR. The basic geometry is illustrated in Fig. 1. A circumferentially guiding defect is located within a periodic medium, which could consist, for example, of a photonic bandgap crystal (PBC) or Bragg layers. This resonator may be viewed as a wraparound waveguide of the type that was recently analyzed using the coupled-wave formalism, ${ }^{4}$ yielding the structure dispersion curve and mode profile. Here we take advantage of the known solution of the straight waveguide to analyze and derive the required index profile for the annular defect resonator.

We consider the case of harmonic time behavior and slab geometry so that there are no variations in the $z$ direction $(\partial / \partial z=0)$. The modal electrical field polarized in the $z$ direction (TE) satisfies the Helmholtz equation, which in cylindrical coordinates is given by

$$
\frac{1}{\rho} \frac{\partial}{\partial \rho}\left(\rho \frac{\partial E}{\partial \rho}\right)+\frac{1}{\rho^{2}} \frac{\partial^{2} E}{\partial \theta^{2}}+k_{0}^{2} n^{2}(\rho) E=0,
$$

where $\rho$ and $\theta$ are the radial and azimuthal coordinates, respectively, and $k_{0}$ is the wave number in vacuum. To transform the annular waveguide to a straight one, we utilize the following conformal transformation ${ }^{5,6}$ :

$$
\rho=R \exp (U / R), \quad \theta=V / R,
$$

where $R$ is an arbitrary parameter. The transformation in Eq. (2) maps a circle in the real plane with radius $R_{0}$ to a straight line located in $U_{0}=R \ln \left(R_{0} / R\right)$.
The structure in Fig. 1A is transformed into a series of straight lines. The wave equation in the $(U, V)$ plane is obtained by transforming Eq. (1):

$$
\frac{\partial^{2} E}{\partial U^{2}}+\frac{\partial^{2} E}{\partial V^{2}}+k_{0}^{2} n_{\mathrm{eq}}^{2}(U) E=0,
$$

where $n_{\mathrm{eq}}(U)=n(U) \exp (U / R)$ is the profile of the refractive index in the $(U, V)$ plane. The inverse transformation of Eq. (2) is given by

$$
U=R \ln (\rho / R), \quad V=\theta R, \quad n(\rho)=n_{\mathrm{eq}}(\rho) R / \rho .
$$

The transformation in Eq. (2) maps the annular waveguide of Fig. 1 into a straight waveguide in the $(U, V)$ plane. Since the requirements for a confined straight Bragg waveguide, and specifically $n_{\mathrm{eq}}(U)$ in the $(U, V)$ plane, are known, ${ }^{4}$ the refractive-index profile $n(\rho)$ in the real plane can be found simply by the inverse transformation in Eqs. (4).

Figure 2 depicts how a Bragg waveguide in the $(U, V)$ plane is transformed to the $(\rho, \theta)$ plane. It should be noted that the gratings in the $(\rho, \theta)$ plane are spatially chirped; i.e., their period changes as a function of the radius $\rho$. In addition, the gratings'

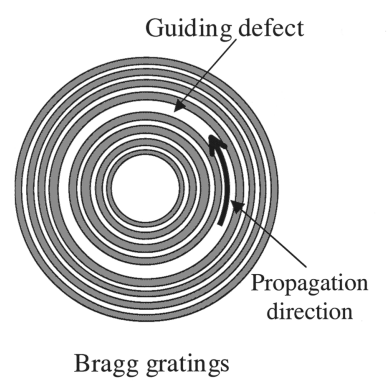

A

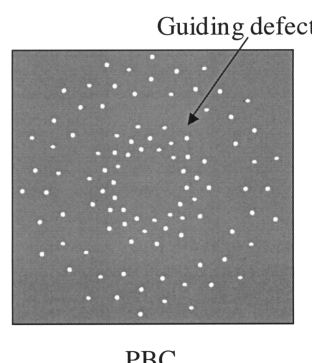

PBC
Fig. 1. Bragg-reflection-based ring resonator. A, Reflection by annular Bragg gratings. B, Realization with an inhomogeneous hole density PBC. 

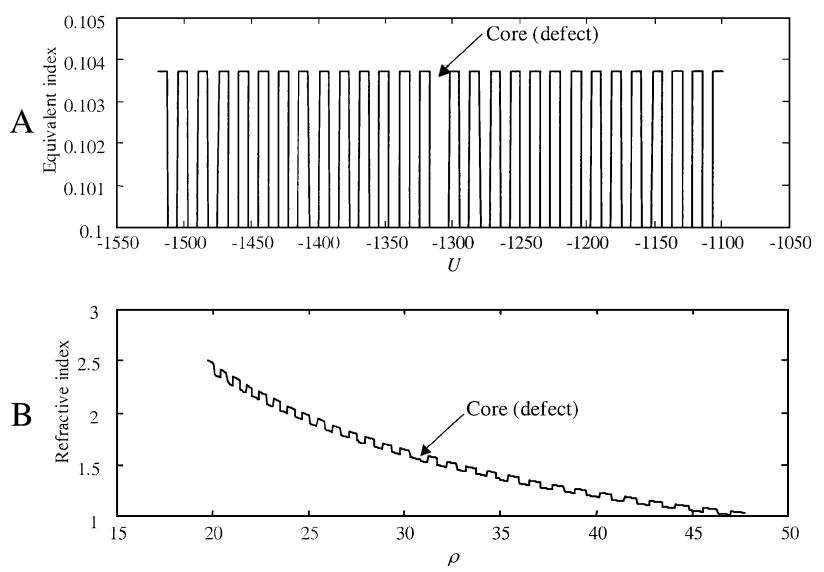

Fig. 2. Refractive-index profile in (A) the $(U, V)$ plane and in $(\mathrm{B})$ the $(\rho, \theta)$ plane.

$$
\begin{aligned}
\left(\Delta n_{\mathrm{eq}}\right)_{l}^{2} & =\frac{1}{b} \int_{b / 2}^{b / 2}\left(n_{\mathrm{eq}}^{2}-\epsilon_{\mathrm{eq}, 0}\right) \exp \left(-i l \frac{2 \pi}{b} V\right) \mathrm{d} V \\
& =\frac{i}{2 \pi l}\left[\left(n_{\mathrm{eq}}^{\max }\right)^{2}-\left(n_{\mathrm{eq}}^{\min }\right)^{2}\right][\exp (-i l \pi)-1],
\end{aligned}
$$

where $n_{\mathrm{eq}}^{\max }$ and $n_{\mathrm{eq}}^{\min }$ are the maximal and minimal refractive indices in the $(U, V)$ plane (see Fig. 2). Unlike the triangular $\mathrm{PBC}$, the perturbation in the case of Bragg layers is purely imaginary and, as a result, the required core width is

$$
W_{\text {Bragg }}=s b / l, \quad s=1,2,3, \ldots
$$

The dispersion relation, however, remains as in Eq. (6). For the case of Bragg layers and $l=1$ the perturbation is $\left(\Delta n_{\mathrm{eq}}\right)_{1}^{2}=1 / \pi i\left[\left(n_{\mathrm{eq}}^{\max }\right)^{2}-\left(n_{\mathrm{eq}}^{\min }\right)^{2}\right]$. The lowestorder solution (mode) of Eq. (3) is given by

$$
\bar{E}(U)=\left\{\begin{array}{ll}
E_{0} \cos \left[\frac{\pi}{b}\left(U-U_{\text {cent }}\right)\right] & \left|U-U_{\text {cent }}\right| \leq \frac{b}{2} \\
E_{0} \cos \left[\frac{\pi}{b}\left(U-U_{\text {cent }}\right)\right] \exp \left[-\kappa_{1}\left(U-U_{\text {cent }}-\frac{b}{2}\right)\right] & \left|U-U_{\text {cent }}\right| \geq \frac{b}{2} \\
E_{0} \cos \left[\frac{\pi}{b}\left(U-U_{\text {cent }}\right)\right] \exp \left[\kappa_{1}\left(U-U_{\text {cent }}+\frac{b}{2}\right)\right] & \left|U-U_{\text {cent }}\right| \leq-\frac{b}{2}
\end{array},\right.
$$

index and index contrast become smaller for larger $\rho$. This effect is caused by the $1 / \rho$ factor multiplying the inverse-transformed index, as seen in Eqs. (4).

Figure 3 depicts the refractive-index profile of an annular waveguide in the $(x, y)$ plane (and the equivalent structure) where the radial confinement is realized by a triangular PBC. As in the case of Bragg-layer reflectors, the required lattice in the real plane is distorted, exhibiting an increasing hole separation for large radii.

Since the mode of this waveguide is known exactly in the $(U, V)$ plane, ${ }^{4}$ the field solution can be readily transformed to the $(\rho, \theta)$ plane. We start with the known solution for the transformed problem in the $(U, V)$ plane:

$$
E(U, V)=\bar{E}(U) \exp (i \beta V),
$$

where $\beta$ is the propagation coefficient. The required core width and the resulting mode profile depend on the specific perturbation used to generate the reflection (Bragg layers, triangular $\mathrm{PBC}$, etc.). As was shown in Ref. 4, for the triangular lattice PBC the required core width is given by $W_{\text {triang }} \mathrm{PBC}=(s+1 / 2) b / l$, where $s=0,1,2, \ldots$, and the dispersion relation is given by

$$
\beta_{\text {triang } \mathrm{PBC}}=\left[k_{0}^{2} \epsilon_{\mathrm{eq}, 0}-(l \pi / b)^{2}\right]^{1 / 2}, \quad l=1,2,3, \ldots,
$$

where $\epsilon_{\mathrm{eq}, 0}$ is the equivalent dielectric constant in the core, $b$ is the perturbation period, and $l$ indicates the Bragg order. For the Bragg-layer structure the perturbation is given by where $\kappa_{1}=2 b\left[\left(n_{\mathrm{eq}}^{\max }\right)^{2}-\left(n_{\mathrm{eq}}^{\min }\right)^{2}\right] / \lambda^{2}$ and $U_{\text {cent }}$ is the coordinate of the center of the guiding channel. The arbitrary transformation parameter $R$ and the value of $U_{\text {cent }}$ determine the radius of the guiding channel in the $(\rho, \theta)$ plane according to $\rho_{\text {cent }}=R \exp \left(U_{\text {cent }} / R\right)$. The radial width of $\Delta \rho$ of the defect is determined similarly.

Figure 4 compares the field profile in the $(U, V)$ plane (Fig. 4A) with that in the $(\rho, \theta)$ plane (Fig. 4B) for a Bragg-layer reflector. The parameters of the structure are defined in the figure caption. The accuracy of the modal solution in Eq. (9) was verified by running a finite-difference time domain simulation of the resonator structure shown on the left-hand side of Fig. 3. As expected, an excellent match was found between the profile calculated by the finite-difference time domain

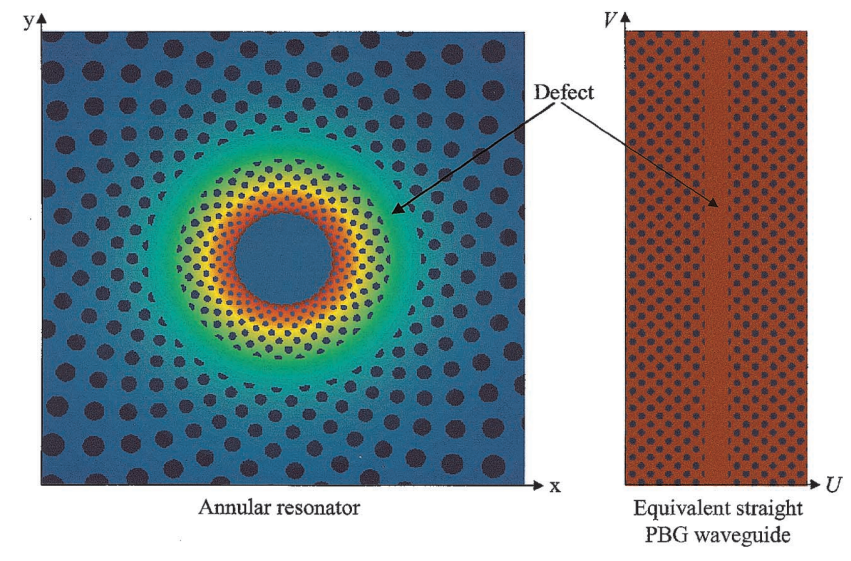

Fig. 3. Refractive index of an annular defect waveguide confined to a triangular $\mathrm{PBC}$ and the equivalent structure. 

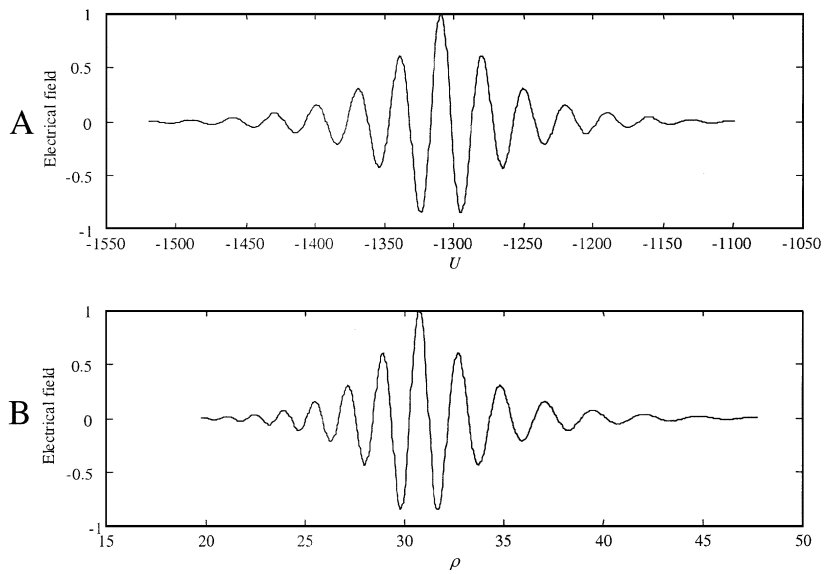

Fig. 4. Field profile in $\mathrm{A}$, the $(U, V)$ plane and in $\mathrm{B}$, the $(\rho, \theta)$ plane. $\quad n_{\mathrm{eq}}^{\min }=0.1, n_{\mathrm{eq}}^{\max }=0.1037, U_{\text {cent }}=-1309.4$, $b=15$.

and the transformed modal solution of the equivalent structure (right-hand side of Fig. 3). The modal field in the circular resonator, $E(\rho, \theta)=\bar{E}(\rho) \exp (i \beta R \theta)$, must satisfy the cyclic boundary condition, $E(\rho, \theta)=$ $E(\rho, \theta+2 \pi)$, and therefore the azimuthal propagation coefficient (the phase shift per revolution) must be an integer:

$$
\begin{aligned}
\beta R & =\left[k_{0}^{2}\left(n_{\mathrm{eq}}^{\min }\right)^{2}-(l \pi / b)\right]^{1 / 2} R=m \\
m & =1,2,3, \ldots
\end{aligned}
$$

For a given $m$ the field $E^{m}(\rho, \theta)$ corresponds to a mode of a new type of resonator formed by the annular defect. The FSR of this resonator is given by

$$
\mathrm{FSR}=\frac{\mathrm{d} v}{\mathrm{~d} m}=\frac{c\left[\left(2 n_{\mathrm{eq}}^{\min } v\right)^{2}-(c l / b)^{2}\right]^{1 / 2}}{\left(2 n_{\mathrm{eq}}^{\min }\right)^{2} R \pi v}
$$

where $c$ is the speed of light in vacuum and $v$ is the optical frequency. The FSR increases if the second term in the numerator is made as small as possible, i.e., if $l=1$ and $b$ is as large as possible. If $b$ is large enough that $2 n_{\mathrm{eq}}^{\mathrm{min}} / \lambda \gg 1 / b$, then the FSR is given by

$$
\mathrm{FSR} \approx \frac{c}{2 n_{\text {Defect }} \pi \rho_{\text {Defect }}},
$$

where $n_{\text {Defect }}$ and $\rho_{\text {Defect }}$ are the index and radius of the defect, respectively. It follows from Eq. (12) that the FSR increases for lower-index defect material. Unlike conventional resonators, where smaller radii require higher core index, the employment of Bragg reflectors allows high confinement of the mode in a low-index defect, regardless of the resonator radius. Therefore a Bragg-reflection-based resonator is expected to have a larger FSR than a conventional resonator of the same radius.

Another important advantage of this structure is that its loss per revolution is determined by the Bragg structure, not by the bending radius (as in conventional ring resonators). By adding more Bragg layers to the large $\rho$ (external) side of the defect, one can make the loss arbitrarily small. In addition, because of the strong Bragg confinement, the defect can be located at any arbitrary radius.

The index profile depicted in Fig. 2A was chosen because it can be solved analytically. However, as can be seen from Fig. $2 \mathrm{~B}$, the required index in the $(\rho, \theta)$ plane might approach larger values at small radii. This could prove to be problematic because the range of refractive indices that can be realized is limited. This problem, however, could be solved easily without sacrificing the advantages of the structure, either by employing asymmetrical gratings in the $(U, V)$ plane or by confining the mode on the small $\rho$ (internal) side of the defect by total internal reflection. It should be emphasized that the confinement method of the mode on the inner side of the resonator has no effect on the resonator bending losses. Moreover, the inner-side confinement is needed only for realizing a single transverse mode and for localizing the intensity in a specific radius. However, the full details of these solutions are beyond the scope of this Letter and will be published separately.

In conclusion, we have presented a new type of microresonator based on Bragg reflection confinement in the radial axis. Closed-form expressions were derived for the electrical field and the dispersion relation. Because of the unique properties of the index profile, the microresonator can be designed to have a very large free spectral range and low loss.

The authors are indebted to the U.S. Air Force Office of Scientific Research (H. Schlossberg) and the Defense Advanced Research Projects Agency (R. Athale) for their support of this work. Useful discussions with S. Mookherjea are acknowledged. J. Scheuer's e-mail address is koby@caltech.edu; A. Yariv's is ayariv@ caltech.edu.

\section{References}

1. A. Yariv, IEEE Photon. Technol. Lett. 14, 483 (2002).

2. B. E. Little, Opt. Lett. 23, 1570 (1998).

3. C. K. Madsen and J. H. Zhao, Optical Filter Design and Analysis: A Signal Processing Approach (Wiley, New York, 1999).

4. A. Yariv, Opt. Lett. 27, 936 (2002).

5. M. Heiblum and J. H. Harris, IEEE J. Quantum Electron. QE-11, 75 (1975).

6. L. Djaloshinski and M. Orenstein, IEEE J. Quantum Electron. 35, 737 (1999). 